\section{Repressors take the tube}

In the developing spinal cord, the ventral neural tube gives rise to motor neurons (MNs) and four classes of interneuron (V0-V3). These are specified in five dorsoventrally arranged progenitor domains ( $\mathrm{pMN}$ and $\mathrm{p} 0-\mathrm{p} 3$ ), which are delineated by the expression of class I and class II homeodomain (HD) genes, including members of the Pax, Nkx, Dbx and Irx families. Their expression patterns are established by graded responses to sonic hedgehog (Shh) signalling from the notochord and floorplate, and the interdomain boundaries are sharpened and maintained by mutual repression between pairs of dorsally expressed class I and ventrally expressed class II proteins. The HD proteins also control neuronal specification by regulating the expression of subtype determinants, and in a new study reported in Cell, Muhr et al. present evidence that this role is an extension of their repressor activity.

Eight of the ten known progenitor HD genes possess a conserved eh 1 domain that recruits co-repressors of the Groucho-TLE (Gro/TLE) family. In an in vitro reporter assay, the authors showed that the repressor functions of class II Nkx proteins depend on the presence of the eh1 domain. They went on to test whether the eh1 domain is also essential in vivo, both for the repression of class I genes and for the promotion of neuronal subtype determinant expression. By electroporating full-length or eh1-deleted Nkx genes into the neural tube of a chick embryo, they showed that misexpression of $\mathrm{Nkx} 2.2$ or Nkx6.1 caused downregulation of class I HD genes (Pax6 and Dbx2 respectively), but only if the eh1 domain was present. Also, misexpression of Nkx6.1 normally induces ectopic expression of the $\mathrm{MN}$ determinant MNR2, but this effect was abolished if eh1 was deleted. The spatiotemporal expression of Gro/TLE co-repressors in the neural tube is consistent with a role in neuronal specification, and the authors showed

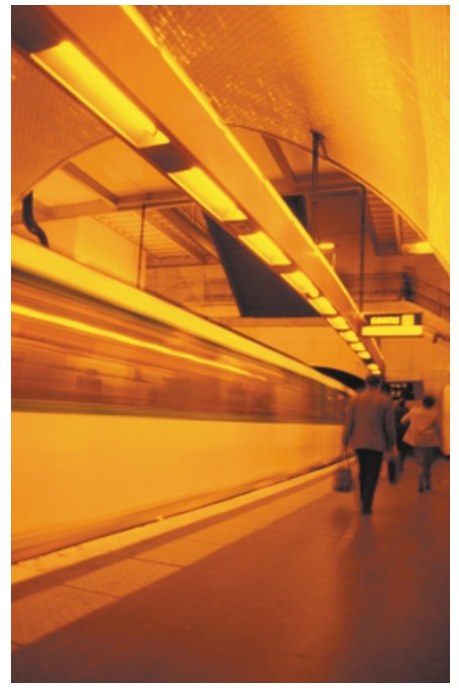

that ectopic expression of Grg5, an endogenous dominant-negative Gro/TLE inhibitor, prevented mutual repression between the class I/class II pairs. Also, although Grg5 expression caused dorsal spreading of the Nkx6.1 expression domain, this did not lead to ectopic specification of MNs, confirming that this too requires Gro/TLE co-repressor activity.

On the basis of these findings, Muhr et al. propose a derepression model for specification of neuronal fate in the neural tube. The pMN domain illustrates how this might work; here, Nkx6.1 directly represses expression of the V1 interneuron determinant and also represses Dbx2 expression, preventing its repression of the gene encoding MNR2. Simultaneously, Pax6, presumably acting through an intermediary repressor because it does not possess an eh1 domain, inhibits expression of the $\mathrm{V} 3$ interneuron determinant. In this way, MNs are specified by derepression of MNR2, while alternative neuronal phenotypes are suppressed. Similar repressive interactions are thought to contribute to neuronal specification in the other progenitor domains.

This model undoubtedly enhances our understanding of neuronal specification in the neural tube, but the question of how the neuronal subtype determinant genes become activated in the first place remains to be answered. Therefore, there is still some way to go before we identify the root of the pathway.

\section{(2) References and links} ORIGINAL RESEARCH PAPER Muhr, J. et al. Groucho-mediated transcriptional repression establishes progenitor cell pattern and neuronal fate in the ventral neural tube. Cell 104, 861-873 (2001) FURTHER READING Jessell, T. M. Neuronal specification in the spinal cord: inductive signals and transcriptional codes. Nature Rev. Genet. 1 20-29 (2000)

ENCYCLOPEDIA OF LIFE SCIENCES Neuronal subtype identity regulation
H I G H L I G H T S

NEURODEGENERATIVE DISORDERS

\section{Fatal attraction}

Huntington's disease is caused by expansion of a polyglutamine tract in the protein huntingtin. Polyglutamine expansion in other proteins can also cause disease: for example, dentatorubral and pallidoluysian atrophy (DRPLA) is related to a polyglutamine expansion in atrophin-1. In Huntington's disease, huntingtin aggregates form in neurons, but the relationship between aggregate formation and neuron death is unclear.

Nucifora et al. looked at the effects of mutant huntingtin and atrophin- 1 on gene transcription mediated by CREBbinding protein (CBP). CREB and CBP mediate the expression of neuronal survival factors, including brain-derived neurotrophic factor (BDNF), and the expression of BDNF is reduced in Huntington's disease. As CBP contains a short polyglutamine tract, Nucifora et al., following earlier suggestions from Perutz, Housman, Fischbeck and others, proposed that the expanded polyglutamine tract in huntingtin might interact with this part of the protein. Indeed, Nucifora et al. found that when CBP and mutant huntingtin were expressed together in neuroblastoma cells, CBP was redistributed away from the nucleus into huntingtin aggregates. By contrast, a modified form of CBP without the polyglutamine tract was not redistributed.

Co-immunoprecipitation experiments showed that CBP and expanded huntingtin interact directly. But how does this interaction cause neuronal toxicity? Nucifora et al. carried out transcription assays on primary cortical neurons and found that mutant huntingtin or atrophin-1 inhibited CBP-mediated gene transcription. They also showed that this effect required abnormal interactions that involve the polyglutamine segments in the proteins, as the use of either normal huntingtin or CBP lacking the polyglutamine tract prevented the inhibition of transcription. Finally, the researchers showed that overexpression of CBP could prevent the death of cells containing the amino-terminal fragment of mutant huntingtin or atrophin-1.

It is still unclear how the two polyglutamine tracts interact. But these results indicate that at least some of the neurotoxic effects of the mutated forms of huntingtin and atrophin-1 probably result from their interaction with CBP, which might inhibit the expression of survival factors such as BDNF. In addition, mutant huntingtin might also interact with other proteins that contain glutamine repeats. So, the interaction between expanded polyglutamine tracts in huntingtin and short glutamine repeats in other proteins might represent a unifying mechanism for polyglutamine toxicity, and a potential target for therapy in diseases such as Huntington's disease and DRPLA.

Rachel Jones

(D) References and links

ORIGINAL RESEARCH PAPER Nucifora, F. C. Jr et al. Interference by huntingtin and atrophin-1 with CBP-mediated transcription leading to cellular toxicity. Science 291, 2423-2428 (2001)

FURTHER READING Gusella, J. F. \& MacDonald, M. E. Unmasking polyglutamine triggers in neurodegenerative disease. Nature Rev. Neurosci. 1, 109-115 (2000) ENCYCLOPEDIA OF LIFE SCIENCES Huntington disease 